DOI 10. 18307/2016. 0617

(C) 2016 by Journal of Lake Sciences

\title{
抚仙湖秋、冬季光衰减特征及其与有色可溶性有机物的关系
}

\author{
周起超 ${ }^{1,2}$, 张运林 $^{2 * *}$, 周永强 ${ }^{2,3}$, 陈毅良 ${ }^{1}$, 秦 江 $^{1}$, 聂菊芬 $^{1}$ \\ (1: 云南省环境科学研究院 (中国昆明高原湖泊国际研究中心) 高原湖泊流域污染过程与管理云南省重点实验室, 昆明 \\ 650034) \\ (2: 中国科学院南京地理与湖泊研究所湖泊与环境国家重点实验室,南京 210008) \\ (3: 中国科学院大学, 北京 100049)
}

\begin{abstract}
摘 要: 为了研究抗仙湖紫外辐射 (UVR) 和光合有效辐射 (PAR) 衰减的时空特征及其与有色可溶性有机物 (CDOM)、悬 浮物 (SS)、浮游植物 (叶绿素 a 表征) 等因子的关系,于 2014 年 10 月 (秋季)、2015 年 1 月 (冬季) 开展现场调查,结果显 示: 秋季不同波长 (段) 的漫射衰减系数 $K_{\mathrm{d}}(305) 、 K_{\mathrm{d}}(340)$ 和 $K_{\mathrm{d}}(\mathrm{PAR})$ 分别为 $1.27 \pm 0.12 、 0.68 \pm 0.11$ 和 $0.32 \pm 0.13 \mathrm{~m}^{-1}$, 冬 季分别为 $1.13 \pm 0.10 、 0.63 \pm 0.07$ 和 $0.36 \pm 0.07 \mathrm{~m}^{-1}$; 秋季 $\mathrm{CDOM}$ 的不同波长吸收系数 $a_{\mathrm{g}}(254) 、 a_{\mathrm{g}}(305)$ 和 $a_{\mathrm{g}}(340)$ 分别为 $4.09 \pm 0.26 、 1.18 \pm 0.09$ 和 $0.57 \pm 0.05 \mathrm{~m}^{-1}$, 冬季分别为 $2.95 \pm 0.24 、 0.61 \pm 0.11$ 和 $0.11 \pm 0.07 \mathrm{~m}^{-1}$, 秋季 $a_{\mathrm{g}}(254) 、 a_{\mathrm{g}}(305)$ 和 $a_{\mathrm{g}}$ (340) 显著高于冬季; 秋季 $K_{\mathrm{d}}(305)$ 显著大于冬季, 这与秋季 (雨季) 较高的 CDOM 丰度、浮游植物生物量 (及 SS 浓度) 有 关. 秋季 $a_{\mathrm{g}}(305) / K_{\mathrm{d}}(305) 、 a_{\mathrm{g}}(340) / K_{\mathrm{d}}(340)$ 均显著高于冬季; 秋季及秋冬季整体而言, $a_{\mathrm{g}}(254)$ 与 $K_{\mathrm{d}}(305) 、 K_{\mathrm{d}}(340)$ 呈 显著正相关, 各多元逐步回归方程中均包含 $a_{\mathrm{g}}(254)$, 说明 CDOM 吸收对 UVR 的衰减有重要贡献. 空间差异方面, 秋季北 部的 $a_{\mathrm{g}}(254) 、 K_{\mathrm{d}}(305)$ 和 $K_{\mathrm{d}}(340)$ 显著高于南部, 冬季南北部无明显差异, 或与雨旱季北岸河流输人的 CDOM 和 SS 的情 况有关. 此外, 浮游植物对 UV-B 衰减的影响和 SS (与 CDOM 的交互作用) 对 UV-A 衰减的影响更在于季节变化方面, 而 影响 UVR、PAR 衰减的各因子的相对贡献有待进一步量化.
\end{abstract}

关键词: 漫射衰减系数; 紫外辐射;真光层;有色可溶性有机物;时空分布;抗仙湖

\section{Spectral attenuation of ultraviolet and visible radiation and its relationship with chromo- phoric dissolved organic matter in autumn/winter in Lake Fuxian, China}

\author{
ZHOU Qichao $^{1,2}$, ZHANG Yunlin ${ }^{2 * *}$, ZHOU Yongqiang ${ }^{2,3}$, CHEN Yiliang $^{1}$, QIN Jiang ${ }^{1}$ \& NIE Jufen ${ }^{1}$ \\ (1: Yunnan Key Laboratory of Pollution Process and Management of Plateau Lake-Watershed, Yunnan Institute of Environmen- \\ tal Science (Kunming China International Research Center for Plateau Lake), Kunming 650034, P.R.China) \\ (2: State Key Laboratory of Lake Science and Environment, Nanjing Institute of Geography and Limnology, Chinese Academy \\ of Sciences, Nanjing 210008, P.R. China) \\ (3: University of Chinese Academy of Sciences, Beijing 100049, P.R.China)
}

\begin{abstract}
To understand the temporal-spatial characteristics of the diffuse attenuation of ultraviolet radiation (UVR) and photosynthetically active radiation (PAR) and its relationship with chromophoric dissolved organic matter( CDOM), suspended solids (SS), chlorophyll-a (Chl.a) and other factors in Lake Fuxian, an oligotrophic deep lake in Yunnan Plateau, we carried out an investigation based on field work in October 2014 ( autumn) and January 2015 ( winter). The results showed that the values of diffuse attenuation coefficient that $K_{\mathrm{d}}(305), K_{\mathrm{d}}(340)$ and $K_{\mathrm{d}}(\mathrm{PAR})$ were $1.27 \pm 0.12 \mathrm{~m}^{-1}, 0.68 \pm 0.11 \mathrm{~m}^{-1}$ and $0.32 \pm 0.13 \mathrm{~m}^{-1}$, respectively in autumn, which were $1.13 \pm 0.10 \mathrm{~m}^{-1}, 0.63 \pm 0.07 \mathrm{~m}^{-1}$ and $0.36 \pm 0.07 \mathrm{~m}^{-1}$, respectively in winter. Meanwhile, the values of absorption coefficient of CDOM that $a_{\mathrm{g}}(254), a_{\mathrm{g}}(305)$ and $a_{\mathrm{g}}(340)$ were $4.09 \pm 0.26 \mathrm{~m}^{-1}, 1.18 \pm 0.09 \mathrm{~m}^{-1}, 0.57 \pm 0.05 \mathrm{~m}^{-1}$, re-
\end{abstract}

* 国家自然科学基金项目 (41601208，41325001)、湖泊与环境国家重点实验室开放基金项目(2016SKL007)、云南省 科技计划项目 (2016RA081) 和云南省应用基础研究计划项目联合资助.2015-11-10 收稿; 2016-01-02 收修改 稿.周起超(1985 ), 男, 博士, 副研究员/博士后; E-mail: qchzhou@ gmail.com.

** 通信作者;E-mail: ylzhang@ niglas.ac.cn. 
spectively in autumn, which were $2.95 \pm 0.24 \mathrm{~m}^{-1}, 0.61 \pm 0.11 \mathrm{~m}^{-1}, 0.11 \pm 0.07 \mathrm{~m}^{-1}$, respectively in winter. The values of of $a_{\mathrm{g}}$ (254), $a_{\mathrm{g}}(305)$ and $a_{\mathrm{g}}(340)$ in autumn were significantly higher than in winter. The values of of $K_{\mathrm{d}}(305)$ in autumn were significantly higher than in winter, which was related with higher CDOM abundance, phytoplankton biomass (and SS concentration) in autumn ( rainy season). Furthermore, the values of $a_{\mathrm{g}}(305) / K_{\mathrm{d}}(305)$ and $a_{\mathrm{g}}(340) / K_{\mathrm{d}}(340)$ in autumn were significantly higher than which in winter; there were significant positive correlations between $a_{\mathrm{g}}(254)$ and $K_{\mathrm{d}}(305), K_{\mathrm{d}}(340)$, and the $a_{\mathrm{g}}(254)$ included in all stepwise regression equations both in autumn and autumn-winter, indicated that the absorption of CDOM was an important factor affecting on the diffuse attenuation of UVR. Moreover, there were obvious differences depend on spatial distribution of $a_{\mathrm{g}}(254), K_{\mathrm{d}}(305)$ and $K_{\mathrm{d}}(340)$ in autumn, the values of $a_{\mathrm{g}}(254), K_{\mathrm{d}}(305)$ and $K_{\mathrm{d}}(340)$ in the north region of the lake was significantly higher than in the south region, whereas there were no significant differences between the north and south regions of $a_{\mathrm{g}}$ (254), $K_{\mathrm{d}}(305)$ and $K_{\mathrm{d}}(340)$ in winter, which might be related with the different river input scenarios of CDOM and SS from the northern shore between autumn ( rainy season) and winter (dry season). In addition, the influences of phytoplankton on UV-B attenuation, the interaction between SS and CDOM on UV-A attenuation were reflected in seasonal changes. However, the relative contribution of pure water, CDOM, phytoplankton and tripton to spectral attenuation (including UVR and PAR) should be quantized in future.

Keywords: Diffuse attenuation coefficient; ultraviolet radiation; euphotic; chromophoric dissolved organic matter; temporalspatial distribution; Lake Fuxian

紫外辐射 (ultraviolet radiation, UVR) 是日光的必有成分, UV-C $(200 \sim 280 \mathrm{~nm})$ 通常被臭氧层和大气层完 全吸收, 到达地面的主要为 UV-B $(280 \sim 320 \mathrm{~nm}$ ) 和 UV-A (320 400 nm) ; UVR 与光合有效辐射 (photosynthetically active radiation, PAR, 400 700 nm) 共同影响着湖泊生态系统的热力学结构、生物地球化学循环、光合 作用与初级生产力、种群动态和群落结构等层面的发展与变化 ${ }^{[1-3]}$. 光辐射在水体中的传输分布主要受纯 水、有色可溶性有机物 ( chromophoric dissolved organic matter, CDOM) 、浮游植物和非色素颗粒物的影响 ${ }^{[1]}$; 其中, CDOM 普遍存在于自然水体, 是重要的光化学、光生物学和生物地球化学物质, 对 UVR 有强烈的吸 收 ${ }^{[1,4]}$. 高海拔清澈型湖泊中, 溶解性有机质 (含 CDOM) 、浮游植物对 UVR 衰减的贡献大 ${ }^{[5-8]}$, 加之二者含量 低, 使其光辐射对流域环境的变化更加敏感; 同时, 海拔高致使所接受的 UVR 强 ${ }^{[9]}$, 较深的 UVR 穿透深度 或使其对湖泊生态系统的影响进一步放大.

抚仙湖是高原深水贫营养湖泊的典型代表, 主导功能为饮用水源地, 保持着良好水质 $(\mathrm{I} \sim \mathrm{II} \text { 类 })^{[10]}$. 然 而, 历史数据已表明, 抚仙湖富营养化和有机污染水平及浮游植物生物量有所升高, 透明度下降, 水质有恶 化趋势 ${ }^{[11-12]}$, 浮游植物功能群 ${ }^{[13]}$ 、底栖动物 ${ }^{[14]}$ 和鱼类 ${ }^{[15]}$ 群落结构等亦已发生变化. 鉴于光对湖泊生态过程 的驱动作用, 要更好地实现对抚仙湖生态环境的保护, 对其光学特征的深人了解是难以回避的. 目前, 涉及 抚仙湖光学特征 (含 CDOM) 的研究并不多 ${ }^{[16-18]}$, 且未涉及时空差异及其影响因子等方面的分析; 秋、冬季不 仅是太阳辐射与温度变化、湖泊热分层变化的重要时期, 还是抚仙湖雨季、旱季的不同代表, 亦是浮游植物 增殖或衰退的关键时期, 以上均可能对 CDOM 的来源、降解以及湖泊光场等产生重要影响. 因此, 本文基于 新近开展的野外调查, 研究了抚仙湖秋、冬两季 UV-B、UV-A、PAR 的衰减特征及其与 CDOM、悬浮物 (suspended solids, SS) 、浮游植物等的关系, 结果既能为抚仙湖积累有关历史数据, 又能为基于湖沼学原理的高 原清澈型深水湖泊的生态环境保护提供支撑.

\section{1 材料与方法}

\section{1 抚仙湖概况}

抚仙湖 $\left(24^{\circ} 21^{\prime} \sim 24^{\circ} 38^{\prime} \mathrm{N}, 102^{\circ} 49^{\prime} \sim 102^{\circ} 57^{\prime} \mathrm{E}\right.$ ), 断陷型湖泊, 地跨云南省玉溪市澄江、江川、华宁三县, 属中亚热带高原半湿润季风气候, 湖泊面积 $216.6 \mathrm{~km}^{2}$, 最大水深 $158.9 \mathrm{~m}$, 平均水深 $95.2 \mathrm{~m}$, 是我国第二深内 陆淡水湖泊; 运行水位 $1720.8 \sim 1722.5 \mathrm{~m}$, 蓄水量 $206.2 \times 10^{8} \mathrm{~m}^{3}$, 占云南九大高原湖泊总蓄水量的 $67.9 \%$, 属 贫营养湖泊 ${ }^{[10]}$.

\section{2 样品采集与指标测定}

在抚仙湖设置 16 个点位 (图 1, $1^{\#} \sim 9^{\#}$ 代表北部, $10^{\#} \sim 16^{\#}$ 代表南部), 于 2014 年 10 月下旬(秋季) 和 
2015 年 1 月下旬 (冬季) 开展调查工作. 各点位均用 PUV-2500 水下剖面辐射仪 (Biospherical Instruments Inc., USA) 测定不同深度不同波长 (段) 的光强, 测定的深度范围为 $0 \sim 3.3 \mathrm{~m}$, 再根据指数拟合计算 UV-B $(305 \mathrm{~nm}) 、 \mathrm{UV}-\mathrm{A}(340 \mathrm{~nm})$ 与 $\operatorname{PAR}(400 \sim 700 \mathrm{~nm})$ 衰减系数 $\left(K_{\mathrm{d}}\right)$, 并计算 $1 \%$ 辐射深度 $\left(Z_{1 \%}\right)^{[16]}$; 用多参数水 质监测仪 YSI 6600 ( Yellow Springs Instruments, USA) 测定水柱水温(WT)、电导率 (EC)、pH 和溶解氧( DO), 由此计算热分层期的温跃层相关指标 ${ }^{[19]}$; 与此同时,采集各点位水下 $0.5 \mathrm{~m}$ 水样,测定其它相关指标.

CDOM 光谱吸收系数:采用经孔径 $0.22 \mu \mathrm{m}$ 的 Millopore 膜过滤的水样在带积分球的 UV-2550 PC 型分 光光度计 (SHIMADZU,Japan) 下测定 200 800 nm 波长范围的吸光度, 然后进行计算、校正得到各波长的吸 收系数 ${ }^{[18]}$, 以 $254 \mathrm{~nm}$ 的吸收系数 $\left(a_{\mathrm{g}}(254)\right)$ 表征 $\mathrm{CDOM}$ 丰度 ${ }^{[20]} ; a_{\mathrm{g}}(\lambda) / K_{\mathrm{d}}(\lambda)$ 为 $\lambda$ 波长时 CDOM 吸收系数 与漫射衰减系数的比例 ${ }^{[16]}$. 溶解性有机碳 (dissolved organic carbon, DOC) 浓度: 水样经 GF/F 滤膜过滤后, 用 TOC-VCPN 仪 (SHIMADZU, Japan) 通过高温燃烧法测定. 总氮 ( TN)、总磷 ( TP)、化学耗氧量 $\left(\mathrm{COD}_{\mathrm{Mn}}\right)$ 、 $\mathrm{SS}$ 、叶绿素 $\mathrm{a}(\mathrm{Chl} . \mathrm{a})$ 浓度的测定参照文献 [21].

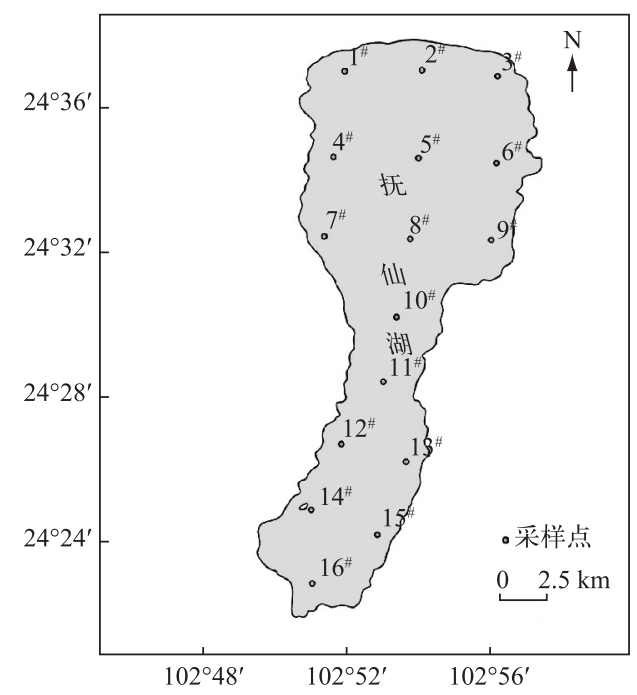

图 1 抚仙湖采样点位置

Fig. 1 Location of sampling sites in Lake Fuxian

\section{3 统计分析}

两独立样本 $\mathrm{T}$ 检验、Pearson 相关性分析及一元线性回归和多元逐步回归均采用 SPSS 22.0 软件完成, $P<0.05$ 表示显著, $P<0.01$ 表示极显著.

\section{2 结果}

\section{1 抚仙湖水柱表层理化参数与湖泊热分层}

调查期间, 抚仙湖秋季水柱表层 WT、EC、TP 浓度、COD $\mathrm{Mn}_{\mathrm{n}}$ DOC 浓度、Chl.a 浓度显著大于冬季, 而 $\mathrm{TN}$ 浓 度显著小于冬季, 秋、冬季 $\mathrm{pH} 、 \mathrm{DO} 、 \mathrm{SS}$ 无显著差异 (表 1). 秋季有明显的热分层现象, 其温跃层上界深度为 $28.64 \pm 1.98 \mathrm{~m}$ 、温跃层下界深度为 $34.07 \pm 2.19 \mathrm{~m}$ 、温跃层厚度为 $5.56 \pm 1.92 \mathrm{~m}$ 、温跃层强度为 $0.79 \pm 0.20^{\circ} \mathrm{C} / \mathrm{m}$; 冬季水温相对均一, 无明显热分层. 由于秋季温跃层的深度高达 $28.64 \pm 1.98 \mathrm{~m}$, 因此在表层的 $3.3 \mathrm{~m}$ 范围内 进行水下光辐射垂直剖面测定不会受湖泊分层的影响,混合层水柱垂直上相对均一.

\section{2 抚仙湖漫射衰减系数与 $1 \%$ 辐射深度}

秋季 UV-B、UV-A 和 PAR 的衰减系数分别为 $1.27 \pm 0.12 、 0.68 \pm 0.11$ 和 $0.32 \pm 0.13 \mathrm{~m}^{-1}$, 冬季 UV-B、UV-A 和 PAR 的衰减系数分别为 $1.13 \pm 0.10 、 0.63 \pm 0.07$ 和 $0.36 \pm 0.07 \mathrm{~m}^{-1}$, 秋季 UV-B 衰减系数显著高于冬季 $(P<$ 0.01 , 表 1$)$, 秋、冬季 UV-A $(P=0.110) 、 P A R(P=0.138)$ 衰减系数的差异则不显著 $($ 表 1$)$; 相应的, 冬季 UV-B 
表 1 抚仙湖水柱表层有关参数

Tab.1 Some parameters in the surface of water column in Lake Fuxian

\begin{tabular}{|c|c|c|c|c|}
\hline \multirow{2}{*}{ 参数 } & \multicolumn{2}{|c|}{ 秋季 $(N=16)$} & \multicolumn{2}{|c|}{ 冬季 $(N=16)$} \\
\hline & 平均值 \pm 标准差 & 范围 & 平均值 \pm 标准差 & 范围 \\
\hline $\mathrm{WT} /{ }^{\circ} \mathrm{C} * *$ & $20.18 \pm 0.15$ & $19.93 \sim 20.40$ & $13.88 \pm 0.09$ & $13.77 \sim 14.06$ \\
\hline $\mathrm{EC} /(\mathrm{mS} / \mathrm{cm}) * *$ & $0.334 \pm 0.00$ & $0.332 \sim 0.335$ & $0.344 \pm 0.00$ & $0.343 \sim 0.346$ \\
\hline $\mathrm{pH}$ & $8.67 \pm 0.14$ & $8.42 \sim 8.94$ & $8.59 \pm 0.15$ & $8.27 \sim 8.91$ \\
\hline $\mathrm{DO} /(\mathrm{mg} / \mathrm{L})$ & $7.43 \pm 0.30$ & $7.01 \sim 7.94$ & $7.69 \pm 0.67$ & $6.54 \sim 8.29$ \\
\hline $\mathrm{TN} /(\mathrm{mg} / \mathrm{L})^{* * *}$ & $0.07 \pm 0.01$ & $0.04 \sim 0.09$ & $0.16 \pm 0.02$ & $0.13 \sim 0.20$ \\
\hline $\mathrm{TP} /(\mathrm{mg} / \mathrm{L})^{* *}$ & $0.044 \pm 0.004$ & $0.040 \sim 0.050$ & $0.039 \pm 0.004$ & $0.030 \sim 0.050$ \\
\hline $\mathrm{COD}_{\mathrm{Mn}} /(\mathrm{mg} / \mathrm{L})^{*}$ & $1.74 \pm 0.13$ & $1.54 \sim 1.99$ & $1.61 \pm 0.15$ & $1.43 \sim 1.90$ \\
\hline $\mathrm{SS} /(\mathrm{mg} / \mathrm{L})$ & $1.65 \pm 0.66$ & $0.49 \sim 2.93$ & $1.25 \pm 0.49$ & $0.40 \sim 2.20$ \\
\hline $\mathrm{DOC} /(\mathrm{mg} / \mathrm{L})^{* * *}$ & $1.96 \pm 0.29$ & $1.79 \sim 2.99$ & $1.46 \pm 0.25$ & $1.31 \sim 2.34$ \\
\hline Chl.a/ $(\mu \mathrm{g} / \mathrm{L})^{* * *}$ & $2.26 \pm 0.21$ & $1.93 \sim 2.60$ & $1.54 \pm 0.53$ & $0.74 \sim 2.61$ \\
\hline$K_{\mathrm{d}}(305) / \mathrm{m}^{-1 * *}$ & $1.27 \pm 0.12$ & $1.11 \sim 1.51$ & $1.13 \pm 0.10$ & $0.89 \sim 1.27$ \\
\hline$K_{\mathrm{d}}(340) / \mathrm{m}^{-1}$ & $0.68 \pm 0.11$ & $0.52 \sim 0.93$ & $0.63 \pm 0.07$ & $0.51 \sim 0.72$ \\
\hline$K_{\mathrm{d}}(\mathrm{PAR}) / \mathrm{m}^{-1}$ & $0.32 \pm 0.13$ & $0.18 \sim 0.68$ & $0.36 \pm 0.07$ & $0.22 \sim 0.47$ \\
\hline$Z_{1 \%}(305) / \mathrm{m}^{* *}$ & $3.65 \pm 0.33$ & $3.05 \sim 4.16$ & $4.10 \pm 0.41$ & $3.62 \sim 5.17$ \\
\hline$Z_{1 \%}(340) / \mathrm{m}$ & $6.95 \pm 1.11$ & $4.95 \sim 8.87$ & $7.45 \pm 0.84$ & $6.38 \sim 8.98$ \\
\hline$Z_{1 \%}(\mathrm{PAR}) / \mathrm{m}$ & $16.14 \pm 5.80$ & $6.76 \sim 26.31$ & $13.18 \pm 2.92$ & $9.74 \sim 20.84$ \\
\hline$a_{\mathrm{g}}(254) / \mathrm{m}^{-1}$ *** & $4.09 \pm 0.26$ & $3.69 \sim 4.60$ & $2.95 \pm 0.24$ & $2.71 \sim 3.44$ \\
\hline$a_{\mathrm{g}}(305) / \mathrm{m}^{-1 * *}$ & $1.18 \pm 0.09$ & $1.02 \sim 1.34$ & $0.61 \pm 0.11$ & $0.44 \sim 0.83$ \\
\hline$a_{\mathrm{g}}(340) / \mathrm{m}^{-1 * *}$ & $0.57 \pm 0.05$ & $0.45 \sim 0.68$ & $0.11 \pm 0.07$ & $0.03 \sim 0.24$ \\
\hline$a_{\mathrm{g}}(305) / K_{\mathrm{d}}(305) / \%^{* *}$ & $92.85 \pm 6.57$ & $81.94 \sim 101.62$ & $54.31 \pm 10.62$ & $35.01 \sim 74.17$ \\
\hline$a_{\mathrm{g}}(340) / K_{\mathrm{d}}(340) / \%^{* *}$ & $85.27 \pm 13.28$ & $63.63 \sim 110.68$ & $18.01 \pm 10.87$ & $5.12 \sim 43.15$ \\
\hline
\end{tabular}

* 表示差异显著 $(P<0.05), * *$ 表示差异极显著 $(P<0.01)$.

的 1\%辐射深度显著高于秋季 $(P<0.01), U V-A 、 P A R$ 的 1\% 辐射深度则无明显差异. 空间差异方面(图 2 4), 秋季北部 UV-B $(P<0.01) 、 \mathrm{UV}-\mathrm{A}(P<0.05)$ 的衰减系数显著高于南部, 对应的, $1 \%$ 辐射深度则为北部显著低 于南部, 南部 PAR 的 $1 \%$ 辐射深度略高于北部, 但无显著性差异; 冬季 UV-B、UV-A 的 $1 \%$ 辐射深度南北部无 明显差异且 UV-B 的 $1 \%$ 辐射深度最深处为湖心附近, 南部 PAR 的 $1 \%$ 辐射深度显著高于北部 $(P<0.05)$.

\section{3 抚仙湖 CDOM 吸收光谱特征与时空分布}

秋、冬季水柱表层 CDOM 吸收光谱表明,200 $225 \mathrm{~nm}$ 时, 冬季的吸收系数高于秋季, 冬季各点位间的差异 大于秋季; $225 ~ 250 \mathrm{~nm}$ 时, 冬季的吸收系数高于秋季(图 5). 秋季 CDOM 在 $254 、 305$ 和 $340 \mathrm{~nm}$ 处的吸收系数 分别为 $4.09 \pm 0.26 、 1.18 \pm 0.09$ 和 $0.57 \pm 0.05 \mathrm{~m}^{-1}$, 冬季 $\mathrm{CDOM}$ 在 $254 、 305$ 和 $340 \mathrm{~nm}$ 处的吸收系数分别为 $2.95 \pm$ $0.24 、 0.61 \pm 0.11$ 和 $0.11 \pm 0.07 \mathrm{~m}^{-1}$, 秋季 $\mathrm{CDOM} 3$ 个波长的吸收系数均显著高于冬季 $(P<0.01$, 表 1$)$. 秋季 $a_{g}(305) / K_{\mathrm{d}}(305) 、 a_{\mathrm{g}}(340) / K_{\mathrm{d}}(340)$ 分别为 $92.85 \% \pm 6.57 \% 、 85.27 \% \pm 13.28 \%$, 冬季分别为 $54.31 \% \pm 10.62 \%$ 、 $18.01 \% \pm 10.87 \%$, 秋季的比值均显著高于冬季的比值, UV-B 的比值显著高于 UV-A 的比值 $(P<0.01$, 表 1$)$. $\mathrm{CDOM}$ 丰度水平空间分布表明 (图 6), 秋季北部显著高于南部 $(P<0.01$ ), 冬季南北部无显著差异, 但中部最低.

\section{4 辐射衰减与各参数相关性}

UV-B、UV-A、PAR 的漫射衰减系数与各参数相关性分析表明, 秋季, $K_{\mathrm{d}}(305)$ 与 $\mathrm{COD}_{\mathrm{Mn}} 、 a_{\mathrm{g}}(254)$ 、 $a_{\mathrm{g}}(305)$ 均呈显著正相关, $K_{\mathrm{d}}(340)$ 与 $a_{\mathrm{g}}(254) 、 a_{\mathrm{g}}(305)$ 均呈显著正相关, $K_{\mathrm{d}}(\mathrm{PAR})$ 与 $a_{\mathrm{g}}(305)$ 呈显著正相关. 冬季, 仅 $K_{\mathrm{d}}(340)$ 与 $\mathrm{TN}$ 呈显著负相关, 其余均无显著相关性 (表 2). 若以秋、冬季为整体进行分析, $K_{\mathrm{d}}(305)$ 与 $\mathrm{TN}$ 呈显著负相关, 与 $\mathrm{TP} 、 \mathrm{COD}_{\mathrm{Mn}} 、 \mathrm{SS} 、 \mathrm{DOC} 、 \mathrm{Chl} . \mathrm{a} 、 a_{\mathrm{g}}(254) 、 a_{\mathrm{g}}(305) 、 a_{\mathrm{g}}(340)$ 均呈显著正相关; $K_{\mathrm{d}}(340)$ 与 $\mathrm{TN}$ 呈显著负相关, 与 $a_{\mathrm{g}}(254) 、 a_{\mathrm{g}}(305)$ 均呈显著正相关. 对 UV-A 、UV-B、PAR 的漫射衰减系数与 CDOM 丰 度、Chl.a、SS 及各参数的交互因子做了多元逐步回归, 结果表明, 秋季 UV-B 衰减主要与 CDOM 丰度及其 SS 
的交互效应有关, UV-A 衰减主要与 CDOM 丰度有关; 秋冬季整体而言, UV-B 衰减与 CDOM 丰度及其与 Chl. $\mathrm{a}$ 和 SS 的交互效应有关, UV-A 衰减与 CDOM 和 SS 的交互效应有关; 冬季, UV-B、UV-A 衰减与检测因子均 无明显关系;不论秋季、冬季或秋冬季为整体,PAR 衰减与检测因子均无明显相关性 (表 3).

表 2 漫射衰减系数与各参数相关性

Tab.2 Pearson correlations between $K_{\mathrm{d}}(\lambda)$ and other parameters in the surface of water column

\begin{tabular}{|c|c|c|c|c|c|c|c|c|c|}
\hline \multirow{2}{*}{ 参数 } & \multicolumn{3}{|c|}{ 秋季 $(N=16)$} & \multicolumn{3}{|c|}{ 冬季( $N=16)$} & \multicolumn{3}{|c|}{ 秋冬季 $(N=32)$} \\
\hline & $K_{\mathrm{d}}(305)$ & $K_{\mathrm{d}}(340)$ & $K_{\mathrm{d}}(\mathrm{PAR})$ & $K_{\mathrm{d}}(305)$ & $K_{\mathrm{d}}(340)$ & $K_{\mathrm{d}}(\mathrm{PAR})$ & $K_{\mathrm{d}}(305)$ & $K_{\mathrm{d}}(340)$ & $K_{\mathrm{d}}(\mathrm{PAR})$ \\
\hline $\mathrm{TN}$ & -0.279 & -0.165 & 0.043 & -0.327 & $-0.558^{*}$ & -0.426 & $-0.595^{\text {*** }}$ & $-0.378^{*}$ & 0.126 \\
\hline TP & 0.145 & 0.152 & 0.241 & 0.340 & 0.183 & -0.176 & $0.427 *$ & 0.272 & -0.002 \\
\hline $\mathrm{COD}_{\mathrm{Mn}}$ & $0.545^{*}$ & 0.436 & 0.191 & 0.033 & -0.125 & -0.494 & $0.456^{*}$ & 0.301 & -0.145 \\
\hline SS & 0.332 & 0.261 & 0.232 & 0.356 & 0.303 & 0.247 & $0.448^{*}$ & 0.342 & 0.154 \\
\hline DOC & 0.025 & -0.031 & -0.102 & 0.230 & 0.119 & -0.255 & $0.441^{*}$ & 0.212 & -0.238 \\
\hline Chl.a & 0.094 & 0.072 & -0.115 & 0.355 & 0.200 & 0.034 & $0.520^{\text {*** }}$ & 0.280 & -0.147 \\
\hline$a_{\mathrm{g}}(254)$ & $0.767^{* * *}$ & $0.674^{\text {*** }}$ & 0.415 & 0.033 & 0.035 & 0.153 & $0.645^{\text {** }}$ & $0.433^{*}$ & -0.054 \\
\hline$a_{\mathrm{g}}(305)$ & $0.635^{* *}$ & $0.621^{*}$ & $0.520 *$ & -0.025 & 0.039 & 0.207 & $0.591^{\text {*** }}$ & $0.380^{*}$ & -0.071 \\
\hline$a_{\mathrm{g}}(340)$ & 0.486 & 0.396 & 0.158 & -0.155 & 0.049 & 0.341 & $0.556^{\text {*** }}$ & 0.335 & -0.135 \\
\hline
\end{tabular}

*表示显著相关 $(P<0.05)$, **表示极显著相关 $(P<0.01)$.

表 $3 K_{\mathrm{d}}(\lambda)$ 与 $a_{\mathrm{g}}(254) 、 C h l . \mathrm{a} 、 \mathrm{SS}$ 及其交互因子的多元逐步回归

Tab.3 Stepwise regression between $K_{\mathrm{d}}(\lambda)$ and $a_{\mathrm{g}}(254)$, Chl.a, SS and their interactive factors

\begin{tabular}{lcccc}
\hline 季节 & $K_{\mathrm{d}}(\lambda)$ & 线性拟合 & $R^{2}$ & $P$ \\
\hline 秋季 & $K_{\mathrm{d}}(305)$ & $y=-0.165+0.323 a_{\mathrm{g}}(254)^{* *}+0.017 a_{\mathrm{g}}(254) \cdot \mathrm{SS}^{*}$ & 0.750 & $<0.001$ \\
$(N=16)$ & $K_{\mathrm{d}}(340)$ & $y=-0.476+0.282 a_{\mathrm{g}}(254)^{* *}$ & 0.454 & $<0.01$ \\
秋冬季 & $K_{\mathrm{d}}(305)$ & $y=0.833+0.084 a_{\mathrm{g}}(254)^{*}+0.007 a_{\mathrm{g}}(254) \cdot \mathrm{Chl} . \mathrm{a} \cdot \mathrm{SS}^{*}$ & 0.498 & $<0.0001$ \\
$(N=32)$ & $K_{\mathrm{d}}(340)$ & $y=0.568+0.016 a_{\mathrm{g}}(254) \cdot \mathrm{SS}$ ** & 0.207 & $<0.01$ \\
\hline
\end{tabular}

表只列出了有显著关系的多元逐步回归方程; * 表示显著 $(P<0.05), * *$ 表示极显著 $(P<0.01)$.

\section{3 讨论}

光辐射在水体中的传输分布主要受纯水、CDOM、浮游植物和非色素颗粒物的影响, 各因子权重变化直 接影响着水下光场结构 ${ }^{[1]}$. Laurion 等 $^{[7]}$ 的研究表明, CDOM 在很大程度上解释了阿尔卑斯山和庇里牛斯山 26 个湖泊间 (海拔 422 2799 m, Chl.a 浓度 $0.25 \sim 3.61 \mu \mathrm{g} / \mathrm{L}$, DOC 浓度 $0.21 \sim 3.50 \mathrm{mg} / \mathrm{L}$ ) UVR 衰减的变化;

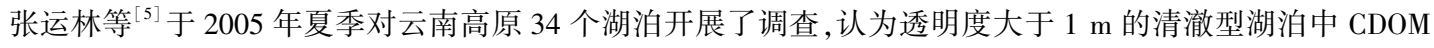
吸收很大程度上决定了 UVR 的影响深度. 2006-2007 年长江中下游平原湖泊的调查结果显示, 其 $a_{\mathrm{g}}(320) /$ $K_{\mathrm{d}}(320) 、 a_{\mathrm{g}}(360) / K_{\mathrm{d}}(360)$ 分别为 $50.7 \% \pm 11.6 \% 、 38.6 \% \pm 12.5 \%$, Zhang 等 ${ }^{[16]}$ 认为这预示着 CDOM 吸收是影 响 UVR 衰减的重要因子之一. 本研究中, 秋季的 $a_{\mathrm{g}}(305) / K_{\mathrm{d}}(305) 、 a_{\mathrm{g}}(340) / K_{\mathrm{d}}(340)$ 与之相当或更高, 且秋 季和秋冬季整体的 UVR 衰减系数与 CDOM 丰度显著正相关, 各逐步回归方程中均包括 $a_{\mathrm{g}}(254)$, 说明 CDOM 吸收在抚仙湖 UVR 的衰减中扮演着重要角色. 基于 CDOM 的吸收光谱可知, 其对 UV-B 的吸收要高 于 UV-A, 而不论秋季或冬季, $a_{\mathrm{g}}(305) / K_{\mathrm{d}}(305)$ 均大于 $a_{\mathrm{g}}(340) / K_{\mathrm{d}}(340)$, 与抚仙湖夏季及云南高原、长江中 下游平原湖泊之前的研究结果一致 ${ }^{[16,18]}$; 不论秋季或秋冬季整体, $K_{\mathrm{d}}(305)$ 与 $a_{\mathrm{g}}(305)$ 均呈极显著正相关, $K_{\mathrm{d}}(340)$ 与 $a_{\mathrm{g}}(340)$ 则无显著相关性, 说明 CDOM 吸收对 UV-B 衰减的影响大于 UV-A. 秋季 $K_{\mathrm{d}}(305)$ 与 $a_{\mathrm{g}}(254) 、 a_{\mathrm{g}}(305)$ 均呈显著正相关, $K_{\mathrm{d}}(340)$ 与 $a_{\mathrm{g}}(254)$ 呈显著正相关, 冬季 $K_{\mathrm{d}}(305) 、 K_{\mathrm{d}}(340)$ 与 $a_{\mathrm{g}}(254)$ 、 $a_{g}(305) 、 a_{g}(340)$ 均无显著相关性, 且未建立相应的逐步回归方程, 说明 CDOM 吸收对秋季 UVR 衰减的影 响大于冬季. 

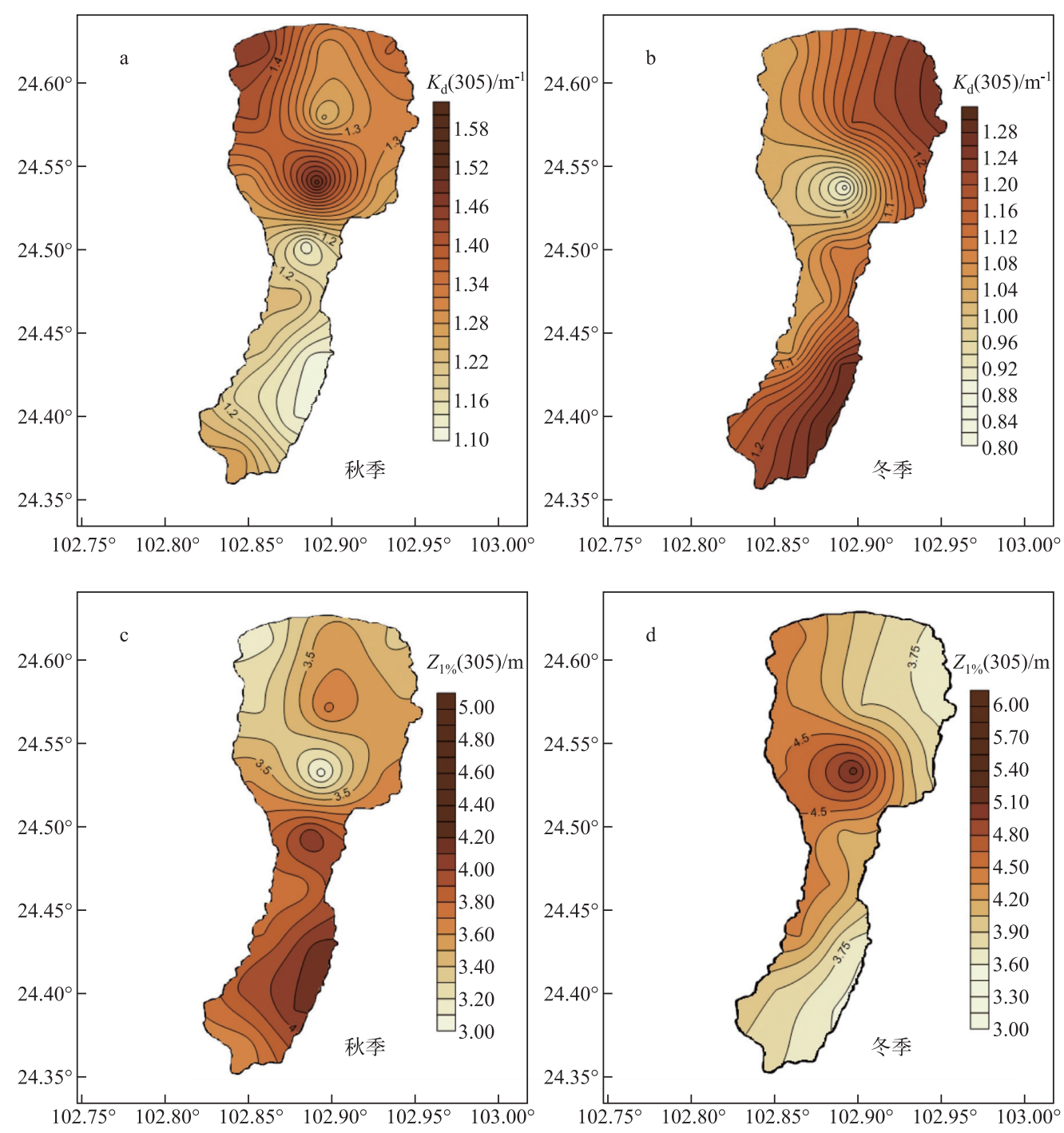

图 2 抚仙湖秋、冬季 UV-B $(305 \mathrm{~nm})$ 衰减系数与 $1 \%$ 辐射深度空间分布

Fig. 2 Spatial distributions of $K_{\mathrm{d}}$ and $Z_{1 \%}$ of UV-B (305 nm) in autumn and winter in Lake Fuxian

CDOM 的吸收光谱特征与水体类型有关 ${ }^{[4]}$, 如湖泊所处的地理位置、营养状态等均可能对其产生影 响 ${ }^{[16,22]}$, 有研究表明云南高原湖泊 CDOM 的吸收整体上小于长江中下游湖泊 ${ }^{[16]}$, 同处云南高原 ${ }^{[18]}$ 或长江 中下游平原 ${ }^{[22-23]}$ 的湖泊之间同样存在差异, 而同一水体的 CDOM 吸收的时空差异已有许多报道 ${ }^{[24-28]}$, 这也 在本研究中得以体现. CDOM 的来源可分为外源的河流输人、降水和内源的沉水植物死亡降解以及沉积物 间隙水的释放 ${ }^{[1]}$, 浮游植物的降解 ${ }^{[27]}$ 、分泌 ${ }^{[29]}$ 以及细菌和水生动物 ${ }^{[30]}$ 等均会产生 CDOM. 本研究中, $a_{\mathrm{g}}$ (254) 与 Chl. a 的回归关系 (Slope $=0.724, R^{2}=0.387, P<0.001, N=32$ ) 表明, 抚仙湖浮游植物对 CDOM 有所 贡献, $a_{\mathrm{g}}(254)$ 与 $\mathrm{COD}_{\mathrm{Mn}}$ 的回归关系 ( Slope $\left.=1.802, R^{2}=0.186, P<0.05, N=32\right)$ 表明, CDOM 是抚仙湖有机污 染的重要组分. 虽然原位产生的 CDOM 会影响其季节变化 ${ }^{[26]}$, 但这并不排除河流输人的主要贡献 ${ }^{[1]}$; 虽然 降水自身携带的 CDOM 对湖体 CDOM 输人的贡献较低 ${ }^{[20]}$, 但雨季的河流输人会携带大量的陆源 $\mathrm{CDOM}^{[24-25,31]}$ 和 $\mathrm{SS}^{[32]}$ 人湖. 抚仙湖北部的澄江县是非点源污染最严重的区域, 其河道径流量占 $63.7 \%$, $\mathrm{TN}$ 、 $\mathrm{TP}$ 输人量分别占 $57.5 \% 、 49.9 \%$, 且雨季 (6-11 月) 远高于旱季 $(12 \text { 月、 } 1-5 \text { 月 })^{[33]}$. 抚仙湖较深且秋季 $(10$ 

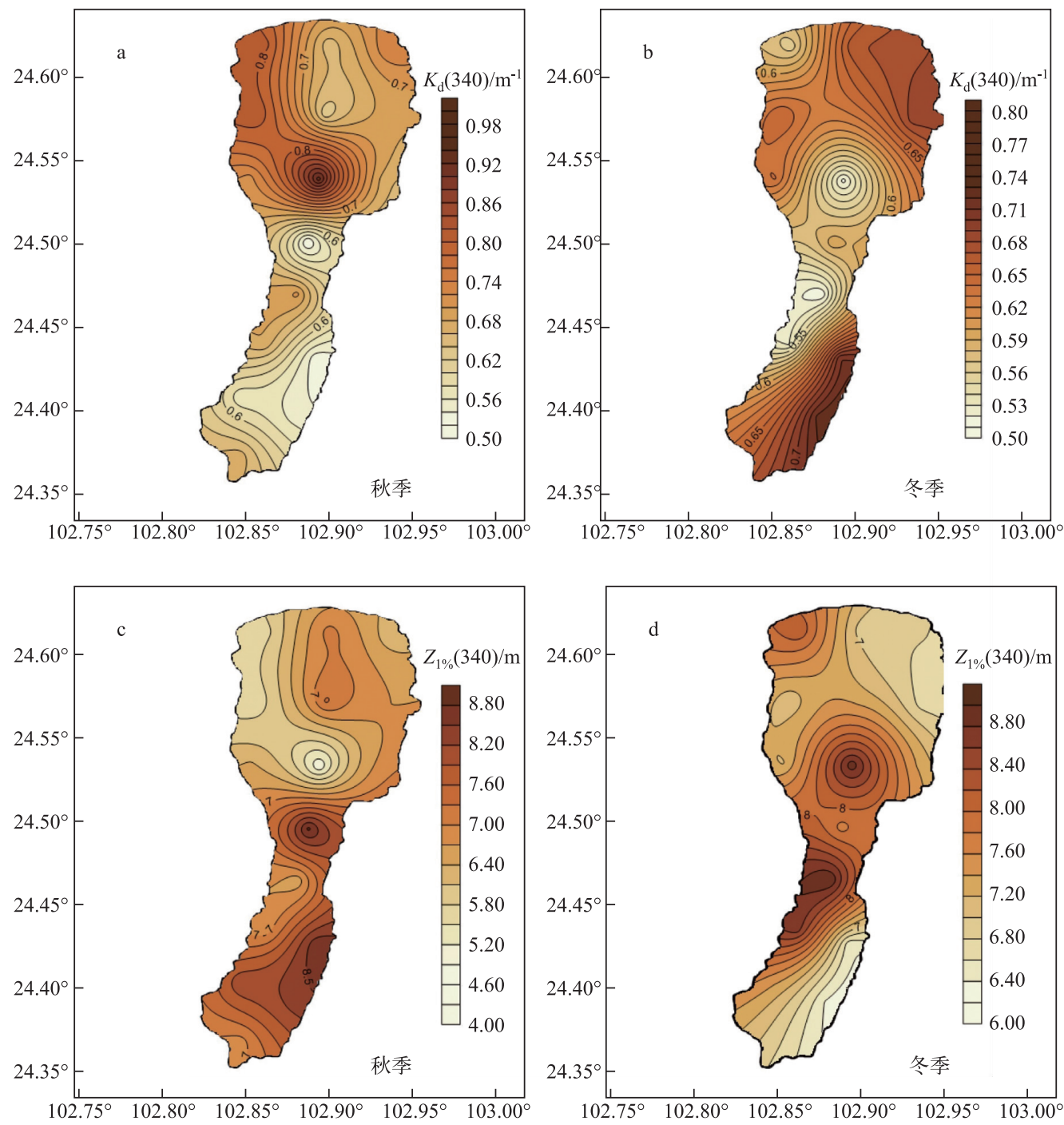

图 3 抚仙湖秋、冬季 UV-A $(340 \mathrm{~nm})$ 衰减系数与 $1 \%$ 辐射深度空间分布

Fig.3 Spatial distributions of $K_{\mathrm{d}}$ and $Z_{1 \%}$ of UV-A $(340 \mathrm{~nm})$ in autumn and winter in Lake Fuxian

月)存在明显的热分层,沉积物间隙水 CDOM 悬浮释放可忽略,故北部 CDOM 丰度显著高于南部,或与南部 UVR 的衰减系数显著低于北部、 $1 \%$ 辐射深度显著高于北部有关. 冬季 (1 月, 旱季) 降水少, 抚仙湖人湖河流 径流量小 ${ }^{[33]}, \mathrm{CDOM}$ 和 SS 的河流输人减少, 水温低浮游植物生物量减少、生理活性降低, 陆源和藻源 CDOM 均随之减少, 加之光降解作用 ${ }^{[34]}$, 故冬季 CDOM 丰度显著低于秋季, 又因湖水的梯度稀释作用 ${ }^{[31]}$, 导致 CDOM 丰度南北部无明显差异且湖心附近最低, 或与 UV-B 的衰减系数最小值、 $1 \%$ 辐深度最大值在湖心有 关. 同时,相关性分析和多元逐步回归结果表明,UV-B 衰减的季节变化与 CDOM 、SS 和浮游植物有关,加之 秋、冬季(雨旱季) 的情景差异 (如前所述), 故秋季 UV-B 衰减系数显著大于冬季. 此外, 本研究显示抚仙湖 $\mathrm{SS}$ 浓度为 $1.45 \pm 0.61 \mathrm{mg} / \mathrm{L} 、 \mathrm{Chl} . \mathrm{a}$ 浓度为 $1.90 \pm 0.54 \mu \mathrm{g} / \mathrm{L} 、$ DOC 浓度为 $1.71 \pm 0.37 \mathrm{mg} / \mathrm{L}$, 暗示清澈型湖泊由于 SS 浓度、DOC 浓度 (CDOM 丰度) 、浮游植物生物量的本底值低, 光辐射特征易受流域有机质输人的影响, 与 有关结果一致 ${ }^{[35-36]}$.

就辐射衰减的其它影响因子而言. 高海拔清澈型湖泊中, 浮游植物对 UVR 衰减的显著影响主要表现在 

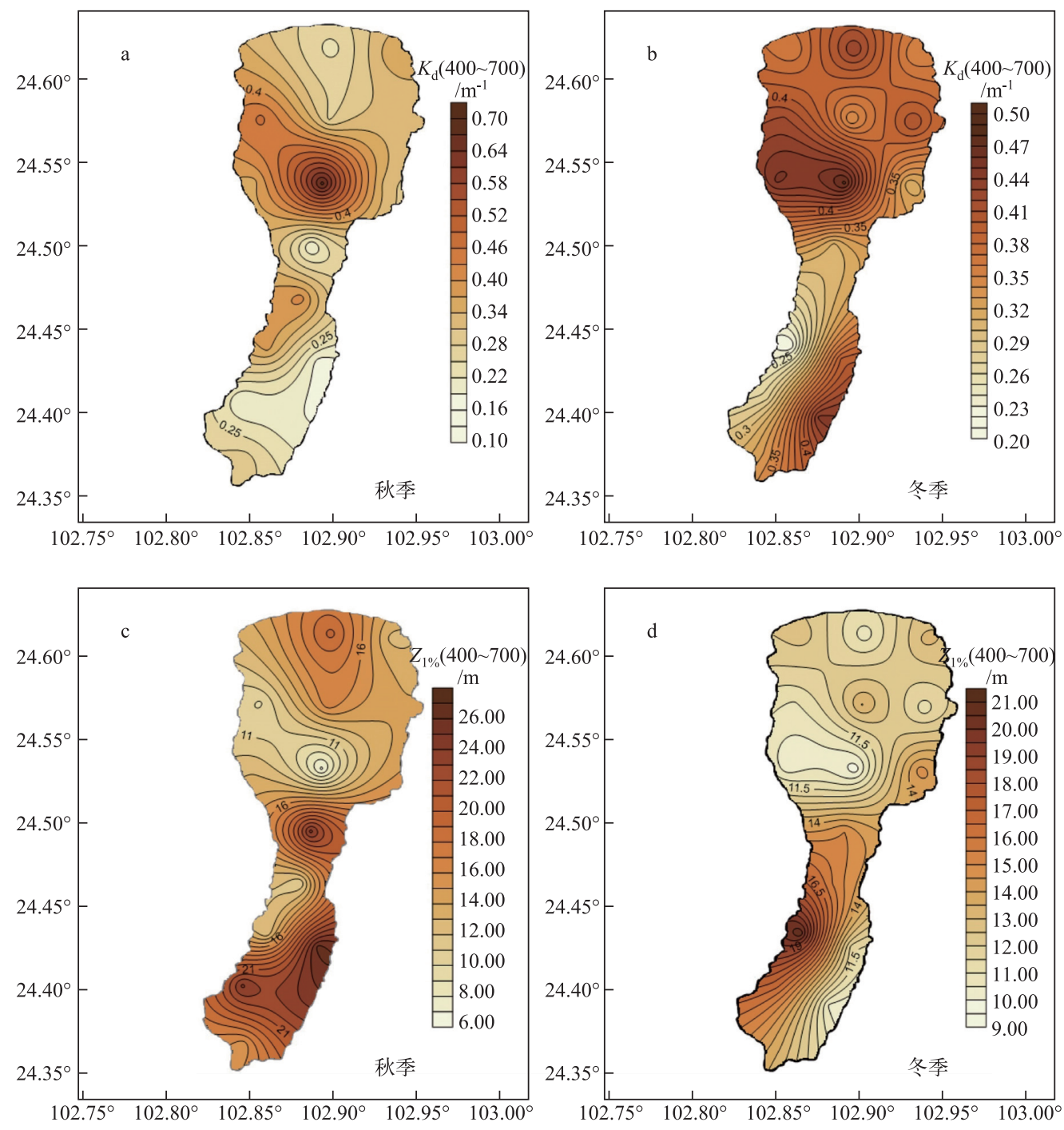

图 4 抚仙湖秋、冬季 PAR (400 700 nm) 衰减系数与 $1 \%$ 辐射深度空间分布

Fig.4 Spatial distributions of $K_{\mathrm{d}}$ and $Z_{1 \%}$ of PAR $(400-700 \mathrm{~nm})$ in autumn and winter in Lake Fuxian

低 DOC 浓度条件下 ${ }^{[7-8]}$, 如阿尔卑斯山 Gossenköllesee 湖紫外穿透深度在时间尺度上的减小与浮游植物 Chl.a 浓度的上升显著相关 ${ }^{[8]}$. 本研究中, 虽然 Chl. a 浓度与 UV-B 的衰减在整体上呈显著正相关, 但同一季节内 并无明显关系, 说明浮游植物的作用主要体现在季节变化上, SS、DOC (不等同于 CDOM) 与之类似; 根据回 归方程可知,SS、Chl.a 对 UV-B 衰减的影响存在与 CDOM 的交互效应, SS 与 CDOM 的交互效应在一定程度 上影响了 UV-A 衰减的季节变化. 此外, 远洋中纯水对光衰减的贡献在波长大于 $440 \mathrm{~nm}$ 时超过了 CDOM、浮 游植物和非色素颗粒物的贡献, 可达 $30 \% \sim 95 \%$ 以上 ${ }^{[4]}$, 而抚仙湖秋冬季 PAR 的衰减与 SS、DOC、Chl.a、 CDOM 丰度等均未表现出明显关系, 且现有数据难以解释冬季 PAR 衰减的南北部差异, 暗示着有必要通过 测定非色素颗粒物、浮游植物等的吸收光谱并量化各组分的相对贡献.

不论 UVR 还是 PAR, 在水柱中的衰减特征依湖泊而不同 ${ }^{[37-38]}$. 抚仙湖是典型的高原清澈型深水湖泊, 接受的 UVR 较长江中下游强, DOC、CDOM、浮游植物和悬浮物 (非色素颗粒物) 等本底浓度低, 光辐射穿透 深度较深, 光辐射特征与浑浊型湖泊明显不同 ${ }^{[16,37]}$. 需要注意的是, 抚仙湖水质的恶化趋势如有机污染水平 


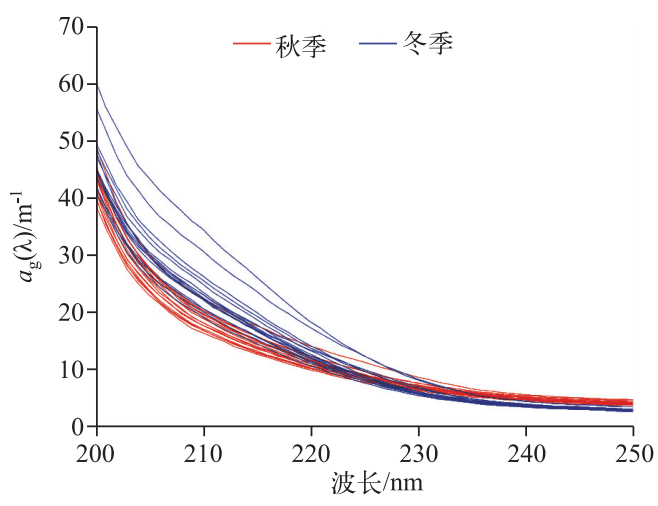

图 5 抚仙湖秋、冬季水柱表层 CDOM 吸收光谱

Fig.5 The absorption spectra of CDOM in the surface water column in autumn and winter in Lake Fuxian
升高、浮游植物生物量升高、透明度降低 等 ${ }^{[11-12]}$, 意味着辐射穿透深度有进一步减小的 可能, 而真光层深度的减小会影响到浅水区沉 水植物的生长, 真光层与混合层深度比的变化 亦会对浮游植物初级生产力、生长与分布等产 生重要影响 ${ }^{[2]}$; 虽然本次调查对抚仙湖光辐射 等特征有了初步了解, 但仍缺乏对其光热特征 全面系统的认识. 此外, 高海拔、清澈型湖泊对 环境变化极为敏感 ${ }^{[6,39-40]}$, 而 UVR 增强、气候变 暖、流域土地利用、污染物输人等的变化, 势必 会对湖泊生态系统的光热特征、生物地球化学 循环、水生生物等方面产生重要影响 ${ }^{[3]}$. 因此, 有必要针对以上方面开展深人研究, 且需注意 不同季节 (及雨季和旱季) 的北部河流的物质 输人情况.
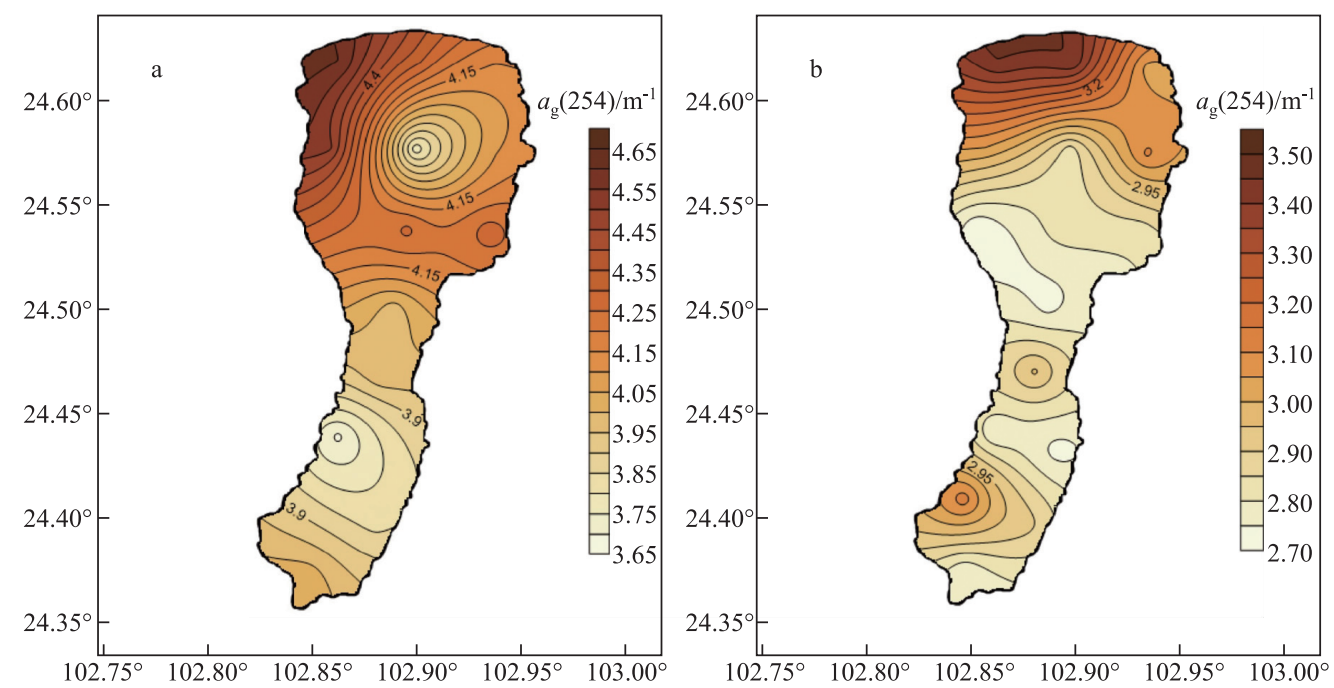

图 6 抚仙湖秋 $(a)$ 、冬 $(\mathrm{b})$ 季 $a_{\mathrm{g}}(254)$ 空间分布

Fig.6 Spatial distributions of $a_{\mathrm{g}}(254)$ of CDOM in autumn (a) and winter (b) in Lake Fuxian

\section{4 结论}

于 2014 年秋季和 2015 年冬季现场获取抚仙湖的光辐射、CDOM 及其它理化数据, 分析 UV-B、UV-A、 PAR 的衰减特征及其与 CDOM 、SS、Chl.a 等因子的关系, 主要结论为: CDOM 吸收对 UVR 衰减具有重要影 响, 对 UV-B 的影响大于 UV-A, 秋季的影响大于冬季; 秋季 UV-B 的衰减系数显著高于冬季, 与秋季(雨季) 较高的 CDOM 丰度、浮游植物生物量 (及县浮物浓度) 有关;秋季北部 UVR 的衰减系数显著高于南部而冬季 南北部无明显差异, 或与雨旱季北部河流 CDOM 和 SS 的输人情况有关; 此外, 浮游植物对 UV-B 衰减的影响 主要体现在季节变化方面, UV-A 衰减的季节变化亦受 SS 与 CDOM 交互作用的影响, 而影响 UVR、PAR 衰 减的各因子的相对贡献有待进一步量化. 


\section{5 参考文献}

[ 1 ] Zhang Yunlin. Progress and prospect in lake optics: A review. J Lake Sci, 2011, 23(4) : 483-497( in Chinese with English abstract). DOI: 10.18307/2011.0401. [张运林. 湖泊光学研究进展及其展望. 湖泊科学, 2011, 23 (4) : 483-497.]

[ 2 ] Kalff J. Limnology: Inland water ecosystems. New Jersey: Prentice Hall, 2002.

[ 3 ] Häder DP, Williamson CE, Wängberg SÅ et al. Effects of UV radiation on aquatic ecosystems and interactions with other environmental factors. Photochemical \& Photobiological Sciences, 2015, 14(1) : 108-26. DOI: 10.1039/c4pp90035a.

[ 4 ] Nelson NB, Siegel DA. The global distribution and dynamics of chromophoric dissolved organic matter. Annual Review of Marine Science, 2013, 5(1) : 447-476. DOI: 10.1146/annurev-marine-120710-100751.

[ 5 ] Zhang Yunlin, Zhang Enlou, Liu Mingliang. Spectral absorption properties of chromophoric dissolved organic matter and particulate matter in Yunnan Plateau lakes. J Lake Sci, 2009, 21(2) : 255-263(in Chinese with English abstract). DOI: 10.18307/2009. 0215. [ 张运林, 张恩楼, 刘明亮. 云南高原湖泊有色可溶性有机物和颗粒物光谱吸收特性. 湖泊科 学, 2009, 21(2): 255-263.]

[ 6 ] Rose KC, Williamson CE, Saros JE et al. Differences in UV transparency and thermal structure between alpine and subalpine lakes: Implications for organisms. Photochemical and Photobiological Sciences, 2009, 8(9) : 1244-1256. DOI: 10. 1039/b905616e.

[ 7 ] Laurion I, Ventura M, Catalan J et al. Attenuation of ultraviolet radiation in mountain lakes: Factors controlling the among-and within-lake variability. Limnology and Oceanography, 2000, 45(6) : 1274-1288. DOI : 10.4319/lo. 2000.45. 6.1274 .

[ 8 ] Sommaruga R, Augustin G. Seasonality in UV transparency of an alpine lake is associated to changes in phytoplankton biomass. Aquatic Sciences, 2006, 68(2) : 129-141. DOI: 10.1007/s00027-006-0836-3.

[ 9 ] Pfeifer MT, Koepke P, Reuder J. Effects of altitude and aerosol on UV radiation. Journal of Geophysical Research: Atmospheres, 2006, 111(D1): D01203. DOI: 10.1029/2005jd006444.

[10] Dong Yunxian, Zhao Lei, Chen Yihui et al. Succession of nine plateau lakes and regulation of ecological safety in Yunnan Province. Ecological Economy, 2015, 31(1): 184-191 (in Chinese with English abstract). [董云仙, 赵否, 陈异晖等. 云南九大高原湖泊的演变与生态安全调控. 生态经济, 2015, 31(1) : 184-191.]

[11] Gao Wei, Chen Yan, Xu Min et al. Trend and driving factors of water quality change in Lake Fuxian $(1980-2011) . J$ Lake Sci, 2013, 25 (5) : 635-642( in Chinese with English abstract). DOI: 10.18307/2013.0503. [ 高伟, 陈岩, 徐敏等. 抚仙湖水质变化(1980-2011 年) 趋势与驱动力分析. 湖泊科学, 2013, 25(5): 635-642.]

[12] Li Yinxi, Liu Hong, Lu Ya et al. Preliminary studies on eutrophication in Fuxian Lake. J Lake Sci, 2003, 15(3) : 285288( in Chinese with English abstract). DOI: 10.18307/2003.0315. [李荫胥, 刘红, 陆娅等. 抚仙湖富营养化初探. 湖 泊科学, $2003, \mathbf{1 5}(3): 285-288$. ]

[13] Dong Jing, Li Genbao, Song Lirong. Historical changes of phytoplankton functional groups in Lake Fuxian, Lake Erhai and Lake Dianchi since 1960s. J Lake Sci, 2014, 26 ( 5) : 735-742 (in Chinese with English abstract). DOI : 10.18307/2014. 0511. [董静, 李根保, 宋立荣. 抚仙湖、洱海、滇池浮游藻类功能群 1960s 以来演变特征. 湖泊科学, 2014, 26(5): 735-742. ]

[14] Xiong Fei, Li Wenchao, Pan Jizheng. Community structure and diversity of macrozoobenthos in Fuxian Lake, a deep plateau lake in Yunnan. Biodiversity Science, 2008, 16(3) : 288-297 (in Chinese with English abstract). DOI: 10.3724/sp.j. 1003.2008.07307. [ 熊飞, 李文朝, 潘继征. 高原深水湖泊抚仙湖大型底栖动物群落结构及多样性. 生物多样性, 2008, 16(3) : 288-297.]

[15] Xiong Fei, Li Wenchao, Pan Jizheng et al. Status and changes of fish resources in Lake Fuxian, Yunnan Province. J Lake Sci, 2006, 18(3) : 305-311 (in Chinese with English abstract). DOI: 10.18307/2006.0318. [熊飞, 李文朝, 潘继征等. 云南抚仙湖鱼类资源现状与变化. 湖泊科学, 2006, 18(3): 305-311.]

[16] Zhang Y, Yin Y, Zhang E et al. Spectral attenuation of ultraviolet and visible radiation in lakes in the Yunnan Plateau, and the middle and lower reaches of the Yangtze River, China. Photochemical and Photobiological Sciences, 2011, 10(4): 469-482. DOI: 10.1039/c0pp00270d. 
[17] Zhang Y, Zhang E, Yin Y et al. Characteristics and sources of chromophoric dissolved organic matter in lakes of the Yungui Plateau, China, differing in trophic state and altitude. Limnology and Oceanography, 2010, 55 (6) : 2645-2659. DOI : $10.4319 /$ lo.2010.55.6.2645.

[18] Zhang Y, Zhang E, Liu M et al. Variation of chromophoric dissolved organic matter and possible attenuation depth of ultraviolet radiation in Yunnan Plateau lakes. Limnology, 2007, 8(3) : 311-319. DOI: 10.1007/s10201-007-0219-z.

[19] Wang Yinzhu, Pu Peimin. Preliminary study on the thermocline in Fuxian Lake. Transactions of Oceanology and Limnolo$g y, 1982,(4): 1-9$ (in Chinese with English abstract). [王银珠, 兴培民. 抚仙湖水温跃层的初步研究. 海洋湖沼通 报, 1982,(4): 1-9.]

[20] Zhang Y, Gao G, Shi K et al. Absorption and fluorescence characteristics of rainwater CDOM and contribution to Lake Taihu, China. Atmospheric Environment, 2014, 98: 483-491. DOI: 10.1016/j.atmosenv.2014.09.038.

[21] Ministry of Environmental Protection of the People's Republic of China, Editorial Board of Water and Wastewater Monitoring and Analysis Methods ed. Water and Wastewater Monitoring and Analysis Methods: 4th edition. Beijing: China Environmental Science Press, 2002(in Chinese). [ 国家环境保护总局《水和废水监测分析方法》编委会. 水和废水监测分 析方法: 第 4 版. 北京: 中国环境科学出版社, 2002.]

[22] Su Wen, Jiang Guangjia, Kong Fanxiang et al. Characteristics of chromophoric dissolved organic matter in inland waters. Resources and Environment in the Yangtze Basin, 2015, 24(1): 114-121 (in Chinese with English abstract). DOI: 10. 11870/cjlyzyyhj201501015. [苏文, 姜广甲, 孔繁翔等. 内陆水体有色溶解有机物的变化特征. 长江流域资源与环 境, 2015, 24(1): 114-121.]

[23] Shi Kun, Li Yunmei, Wang Qiao et al. Similarities and differences in absorption characteristics and composition of CDOM between Taihu Lake and Chaohu Lake. Chinese Journal of Environmental Science, 2010, 31(5) : 1183-1191(in Chinese with English abstract). [施坤, 李云梅, 王桥等. 太湖、巢湖水体 CDOM 吸收特性和组成的异同. 环境科学, 2010, 31(5): 1183-1191.]

[24] Zhou Y, Zhang Y, Shi K et al. Dynamics of chromophoric dissolved organic matter influenced by hydrological conditions in a large, shallow, and eutrophic lake in China. Environmental Science and Pollution Research, 2015, 22 (17) : 1299213003. DOI : 10.1007/s11356-015-4556-x.

[25] Zhang Y, Yin Y, Feng L et al. Characterizing chromophoric dissolved organic matter in Lake Tianmuhu and its catchment basin using excitation-emission matrix fluorescence and parallel factor analysis. Water Research, 2011, 45(16) : 51105122. DOI: 10.1016/j.watres.2011.07.014.

[26] Del Castillo CE, Miller RL. Horizontal and vertical distributions of colored dissolved organic matter during the Southern Ocean Gas Exchange Experiment. Journal of Geophysical Research: Oceans, 2011, 116 ( C4): C00F07. DOI: 10. 1029/2010jc006781.

[27] Zhang Y, Yin Y, Liu X et al. Spatial-seasonal dynamics of chromophoric dissolved organic matter in Lake Taihu, a large eutrophic, shallow lake in China. Organic Geochemistry, 2011, 42 (5) : 510-519. DOI: 10.1016/j. orggeochem. 2011. 03.007 .

[28] VaiČiūté D, Bresciani M, Bartoli M et al. Spatial and temporal distribution of coloured dissolved organic matter in a hypertrophic freshwater lagoon. Journal of Limnology, 2015, 74(3) : 572-583. DOI : 10.4081/jlimnol.2015.1176.

[29] Castillo CR, Sarmento H, Álvarez-Salgado XA et al. Production of chromophoric dissolved organic matter by marine phytoplankton. Limnology and Oceanography, 2010, 55(1) : 446-454. DOI: 10.4319/lo.2010.55.1.0446.

[30] Ortega-Retuerta E, Frazer TK, Duarte CM et al. Biogeneration of chromophoric dissolved organic matter by bacteria and krill in the Southern Ocean. Limnology and Oceanography, 2009, 54 (6) : 1941-1950. DOI: 10.4319/lo.2009.54.6.1941.

[31] Feng Longqing, Liu Mingliang, Zhang Yunlin et al. Upstream contributions to the chromophoric dissolved organic matter in the Taihu Lake during summer rainy seasons. Advances in Water Science, 2011, 22(1) : 104-111(in Chinese with English abstract). DOI : 32-1309/p.20110115.2241.009. [冯龙庆, 刘明亮, 张运林等. 夏季丰水期河流输人对太湖有色可溶 性有机物的贡献. 水科学进展, 2011, 22(1): 104-111.]

[32] Zhang Y, Shi K, Zhou Y et al. Monitoring the river plume induced by heavy rainfall events in large, shallow, Lake Taihu using MODIS 250 m imagery. Remote Sensing of Environment, 2016, 173: 109-121. DOI: 10.1016/j.rse.2015.11.020.

[33] Xia Tianxiang, Pan Jizheng, Liu Xuehua et al. Non-point source pollution characteristics in Fuxianhu lake watershed and 
variation law of N and P in lake water. Journal of Agro-environment Science, 2008, 27(4) : 1340-1345( in Chinese with English abstract). [夏天翔, 潘继征, 刘雪华等. 抚仙湖水体 N、P 变化及其非点源污染特征. 农业环境科学学报, 2008, 27(4) : 1340-1345.]

[34] Zhang Y, Liu M, Qin B et al. Photochemical degradation of chromophoric-dissolved organic matter exposed to simulated UV-B and natural solar radiation. Hydrobiologia, 2009, 627 (1) : 159-168. DOI : 10.1007/s10750-009-9722-z.

[35] Williamson CE, Stemberger RS, Morris DP et al. Ultraviolet radiation in North American lakes: Attenuation estimates from DOC measurements and implications for plankton communities. Limnology and Oceanography, 1996, 41(5) : 1024-1034. DOI: $10.4319 /$ lo.1996.41.5.1024.

[36] Nevalainen L, Luoto TP, Rantala, MV et al. Role of terrestrial carbon in aquatic UV exposure and photoprotective pigmentation of meiofauna in subarctic lakes. Freshwater Biology, 2015, 60(11) : 2435-2444. DOI: 10.1111/fwb.12670.

[37] De Lange HJ. The attenuation of ultraviolet and visible radiation in Dutch inland waters. Aquatic Ecology, 2000, 34(3) : 215-226. DOI: 10.1023/A : 1009943211779 .

[ 38 ] Rose KC, Hamilton DP, Williamson CE et al. Light attenuation characteristics of glacially-fed lakes. Journal of Geophysical Research: Biogeosciences, 2014, 119(7) : 2014JG002674. DOI: 10.1002/2014jg002674.

[39] Scully N, Vincent WF, Lean DRS et al. Implications of ozone depletion for surface-water photochemistry: Sensitivity of clear lakes. Aquatic Sciences, 1997, 59(3) : 260-274. DOI : 10.1007/bf02523277.

[40] Catalan J, Camarero L, Felip M et al. High mountain lakes: Extreme habitats and witnesses of environmental changes. Limnetica, 2006, 25(1) : 551-584. DOI: 10.1016/j.jelekin.2009.07.004. 